\title{
Déterminisme de la surface des vaisseaux du bois des chênes indigènes (Quercus robur $L$, Quercus petraea Liebl). Effet individuel, effet de l'appareil foliaire, des conditions climatiques et de l'âge de l'arbre
}

\author{
F Huber \\ INRA, station de recherches sur la qualité des bois, 54280 Champenoux, France
}

(Reçu le 17 juin 1992; accepté le 6 avril 1993)

\begin{abstract}
Résumé - Les résultats de 2 études concernant le déterminisme de la surface individuelle des vaisseaux du bois initial de chênes indigènes de France (Quercus robur $L$ et Quercus petraea Liebl) sont rapportés : I'une a été menée sur 15 jeunes chênes pédonculés ( 3 ans) de provenance "Vallée de l'Adour', sur lesquels la surface individuelle des vaisseaux de la première rangée formée au printemps a été mesurée; l'autre a été menée sur 37 chênes adultes (200 ans environ, sans distinction d'espéces) sur lesquels l'ensemble des vaisseaux du bois initial a été pris en considération : sur 17 années pour le bois juvénile et 20 années dans le bois adulte. Les résultats obtenus peuvent se résumer de la façon suivante :

- chez les jeunes plants : l'effet de l'âge cambial est hautement significatif ; les vaisseaux de l'année qui suit celle de l'ablation des feuilles ont une surface moindre comparée à celle du lot témoin ;

- chez les arbres adultes : dans le bois juvénile, les variations dues à l'arbre lui-même et à l'âge cambial sont de même importance ; elles expliquent à elles deux $67 \%$ de la variabilité totale. Dans le bois adulte, l'effet de l'arbre à lui seul explique $60 \%$ de la variabilité totale. L'effet de l'année calendaire, bien que significatif, n'explique que $4 \%$ de la variabilité totale ; une corrélation faible mais significative existe entre la surface individuelle des vaisseaux et l'une des données climatiques étudiées (températures maximales des mois d'automne précédant la formation des vaisseaux). Aucune relation significative entre la surface individuelle des vaisseaux et la largeur des cernes n'a été mise en évidence dans le bois adulte (niveaux inter- et intra-arbre).
\end{abstract}

Quercus petraea / Quercus robur / anatomie / bois initial / vaisseaux / variabilité

Summary - Determination of the area of vessels in the earlywood of indigenous oaks in France (Quercus robur L, Quercus petraea Liebl). Effects related to individual trees, foliar cover, climatic conditions and cambial age. The results of 2 studies on the individual area of vessels in the earlywood of indigenous Oaks in France (Quercus robur $L$ and Quercus petraea Liebl) are reported: one was carried out on 15 young Pedunculate oaks ( 3 y old) from the Adour Valley, in 
which the vessels of the first row formed in early spring, were measured; the trees had been subjected to 3 defoliation treatments; the other was carried out on 37 adult Oaks (about $200 \mathrm{yr}$ old, without distinguishing the species) in which all the vessels of the earlywood were measured: for $17 \mathrm{yr}$ for the juvenile wood and for $20 \mathrm{yr}$ for the adult wood. The results can be summarised as follows: in young plants, the effect of cambial age is highly significant and the vessels formed in the year following defoliation have a smaller area than those of the control; in the juvenile wood of adult trees the individual effect and the cambial age of the tree are equally important, together they explain $67 \%$ of the total variability. There is a low but significant correlation between the individual area of the vessels that were taken into account, and one of the climatic results. No relationship between vessel area and ring in the adult wood have been observed (inter and intratree level).

\section{Quercus petraea / Quercus robur / anatomy / early wood / vessels / variability}

\section{INTRODUCTION}

Ainsi que l'a souligné Polge (1984), la variabilité anatomique du bois de chêne indigène ( $Q$ robur $L$ et $Q$ petraea Liebl) est considérable.

Cette variabilité est probablement à l'origine des propriétés technologiques variées et, par voie de conséquence, des emplois divers qu'il est fait de ce matériau.

Ces remarques ont conduit à 2 types de recherche :

- impact de cette anatomie sur les propriétés technologiques, sur les défauts et sur l'aspect esthétique de ce bois ;

- déterminisme de cette anatomie avec pour objectif de tenter de la maîtriser notamment par voie génétique.

Parmi les éléments anatomiques, les vaisseaux du bois initial sont apparus comme importants.

Citons à ce propos quelques références.

Marchal (1983) a montré que le diamètre des vaisseaux est corrélé de façon significative et positive à l'écart type de l'épaisseur des placages tranchés.

Pechmann et Aufsess (1973) ont fixé à $0,4 \mathrm{~mm}$ le diamètre maximal des vaisseaux pour l'obtention d'un placage de qualité.
En 1986, Savill a mis en évidence le fait que la surface moyenne des vaisseaux du bois initial était le seul paramètre corrélé de façon positive et significative à la présence de fente dans les grumes, alors qu'aucun des autres paramètres pris en considération (nombre de vaisseaux par unité de surface, largeur du bois initial, largeur de cerne et largeur des rayons ligneux) ne permettait de différencier de façon significative une population d'arbres à fentes fréquentes d'une population d'arbres exempts de ce défaut.

Ces résultats ont été corroborés par les travaux de Cinotti (1990) qui a montré lui aussi que la taille des vaisseaux était parmi les caractéristiques anatomiques celle qui différenciait le plus nettement les arbres gélivés des arbres sains.

Avant même de soupçonner le rôle que pouvaient avoir les vaisseaux sur les propriétés du bois, certains facteurs avaient été retenus comme pouvant expliquer la variation de la taille de cet élément.

i) L'environnement : les travaux d'Eckstein et Frisse (1979) ont montré que le climat expliquait une part importante de la variabilité de la taille des vaisseaux. Ces résultats sont contrecarrés par les travaux de Savill et Mather (1988, communication personnelle). 
Cependant ces auteurs ont montré, comme Flechter (1975), que pour les années dont le climat est "exceptionnel» la taille maximale des vaisseaux est faible comparée à celle des années dites normales.

ii) L'appareil foliaire : l'influence exercée par les feuilles sur la formation du bois a fait l'objet d'études aux résultats parfois controversés.

D'après Gradwell (1974), il semble que la mise en place du bois initial et par voie de conséquence des vaisseaux soit conditionnée par la présence des feuilles jusqu'en automne. Huber (1982) montrait que l'élimination des éléments chlorophylliens par les hannetons, c'est-à-dire dès le débourrement printanier, était sans influence sur la proportion des vaisseaux du bois initial et sans influence sur l'accroissement de ce dernier. En revanche, l'accroissement du bois final était moindre les années de défoliaison. Malphettes (1990) n'observe pas de réelle diminution de production ligneuse les années d'attaque de bombyx. Mais il faut noter que ces insectes attaquent les feuilles lorsque cellesci sont déjà bien formées et n'ont alors peut-être plus un très grand rôle sur la formation du bois.

iii) L'âge du bois compté par rapport à la moelle : ce sont les travaux de Gasson (1984, 1987) qui, les premiers, ont mis en évidence le fait que, durant la phase juvénile, la taille des vaisseaux augmentait de façon linéaire avec l'âge cambial. Par la suite ces résultats ont été confirmés par Savill et Mather (1988, communication personnelle).

iv) L'individu lui-même : cette variabilité avait d'ailleurs été soupçonnée pour d'autres paramètres que les vaisseaux (Polge et Keller, 1973).

En comparant les 2 espèces de chêne ( $Q$ petraea Liebl et $Q$ robur $L$ ), Savill et Mather (1988, communication personnelle) ont montré que le rapport des 2 diamètres (tangentiel et radial) des vaisseaux ne différait pas entre les 2 espèces, mais qu'en revanche à l'intérieur des espèces il y a un effet de l'individu hautement significatif qui, en ce qui les concerne, expliquait $23 \%$ de la variation totale. Des travaux plus récents de Kanowski et al (1991) ont mis en évidence l'héritabilité de la surface moyenne des vaisseaux très élevée.

Il est évident que, si l'importance technologique de la taille des vaisseaux du bois des chênes indigènes était telle qu'elle rendrait incontournable leur prise en compte en sélection, il est au préalable nécessaire de bien en connaître le déterminisme.

Face aux propos parfois contradictoires des auteurs, et en absence de travaux concernant le déterminisme de la taille des vaisseaux, nous avons entrepris différentes études dont nous rapportons ici les résultats.

La première a été conduite sur des plants de chêne pédonculé ( $Q$ robur $L$ ) de provenance connue: vallée de l'Adour. Pour ces travaux qui concernaient l'étude de l'effet de l'appareil foliaire sur les vaisseaux de jeunes plants âgés de 3 ans, nous nous sommes affranchis de l'effet de la concurrence, du climat, etc, dont il est parfois difficile de faire la part lorsque l'on considère des arbres adultes, en travaillant en pépinière.

La seconde étude nous a permis de nous intéresser à la variabilité individuelle, à l'effet de l'âge cambial et de la largeur de cerne sur la taille des vaisseaux dans le bois juvénile et dans le bois adulte des mêmes arbres. De plus sur le bois adulte, nous avons testé l'effet du climat.

L'ensemble de cette étude est considéré comme une première étape en vue d'envisager la possibilité d'une sélection portant sur les propriétés du bois de chêne. 


\section{MATÉRIEL ET MÉTHODES}

Nous avons étudié la surface des vaisseaux du bois initial de chênes indigènes sur de jeunes plants et sur des arbres adultes.

Le tableau I présente les points traités pour chacune de ces 2 expériences.

Toutes les mesures ont été réalisées à l'aide d'un analyseur d'image en utilisant des programmes spécifiques mis au point à la station de recherches sur la qualité des bois (Aubert, 1987; Hofmann, 1987). L'ensemble des données a été analysé à l'aide des modules de calcul statistique du logiciel Statitcf.

Les tests statistiques, analyse de variance (test $F$ ) et corrélation ( $r$ ) ont été mis en œuvre sur la moyenne arithmétique de la surface des vaisseaux calculée cerne par cerne pour chaque individu.

\section{Expérience sur jeunes plants : variabilité individuelle et effet de l'appareil foliaire}

\section{Matériel végétal et traitement}

L'étude a été menée uniquement sur des chênes pédonculés ( $Q$ robur $L$ ). Ces plants, issus de glands de provenance de la vallée de l'Adour, ont été élevés en pépinière (pépinière d'Amance, Meurthe-et-Moselle).

$\mathrm{Au}$ cours de la $2^{\mathbf{e}}$ année, à la mi-août, 15 plants ont été sélectionnés et répartis en 3 lots de 5 plants dont l'aspect, la taille (hauteur et diamètre du collet) étaient comparables à l'intérieur de chaque lot et entre les lots.

A cette même date, des traitements différents ont été appliqués sur chacun de ces lots. Les traitements ont été les suivants :

- 1er traitement : les plants ont été totalement défoliés ;

$-2^{e}$ traitement : les plants ont été partiellement défoliés ; la moitié des feuilles, en nombre, a été enlevée.

L'ablation a été réalisée de façon homogène sur toute la longueur de la tige.

Ceci est d'autant plus vrai que dans le cas de ce $2^{e}$ traitement, comme dans le cas des 2 autres, nous n'avons observé aucune vague de croissance en hauteur après la date des différents traitements de défoliaison ;

$-3^{e}$ traitement : les plants ont été conservés intacts (lot témoin).

Au cours du mois de novembre de leur $3^{e}$ année de végétation, c'est-à-dire après une saison en pépinière où ils n'ont subi aucun traitement et ceci quel que soit le lot, les 15 plants ont été arrachés aux fins de mesures.

\section{Mesures réalisées}

Les mesures ont été réalisées à partir de coupes microscopiques de $12 \mu \mathrm{m}$ d'épaisseur, pratiquées transversalement dans un fragment de tige prélevé à $3 \mathrm{~cm}$ au-dessus du collet.

Pour chaque plant, nous avons mesuré 20 vaisseaux par année de végétation soit 900 au total (20 vaisseaux par année de végétation $\times 3$ années de végétation $x 5$ plants par traitement $x$ 3 traitements).

La limite entre le bois initial et le bois final n'est pas bien marquée dans le bois des 3 années les plus proches de la moelle comme elle l'est dans un bois d'âge cambial plus élevé (fig 1).

C'est pourquoi, afin d'éviter une erreur dans la délimitation de chacun des bois (bois initial bois final), nous n'avons mesuré que les vaisseaux de la $1^{\text {re }}$ rangée, c'est-à-dire ceux adjacents au bois final de l'année précédente ou au xylème primaire pour la $1^{\text {re }}$ année cambiale.

\section{Expérience sur arbres adultes : variabilité individuelle, effet de la largeur de cerne, et de l'année calendaire}

\section{Matériel végétal et méthode}

L'étude a été menée sur les chênes des forêts de Bride et Saint-Jean (Moselle), sans distinction d'espèces ( $Q$ robur $L$ et $Q$ petraea Liebl).

Sur 37 arbres, arrivés à l'âge d'exploitabilité 200 ans environ), nous avons prélevé un fragment de bois à la base de la bille, en évitant le bois de l'empattement, soit à une hauteur approximative de $1 \mathrm{~m}$ à 1,30 $\mathrm{m}$ à partir du sol. 
À partir de radiographies de barrettes de $1,5 \mathrm{~mm}$ d'épaisseur (Polge, 1966), nous avons mesuré la surface des vaisseaux du bois initial ainsi que la largeur des accroissements annuels.

Ces mesures ont été réalisées d'une part sur les 20 derniers cernes les plus proches de l'écorce (année 1960 à 1979 inclusivement), et d'autre part sur les 17 années les plus proches de la moelle en excluant les 3 premières années, pour les raisons évoquées au paragraphe "Mesures réalisées".

II faut rappeler que, dans cette étude portant sur les chênes adultes, nous n'avons pas distingué les chênes pédonculés des chênes rouvres qui se côtoient dans ces forêts.
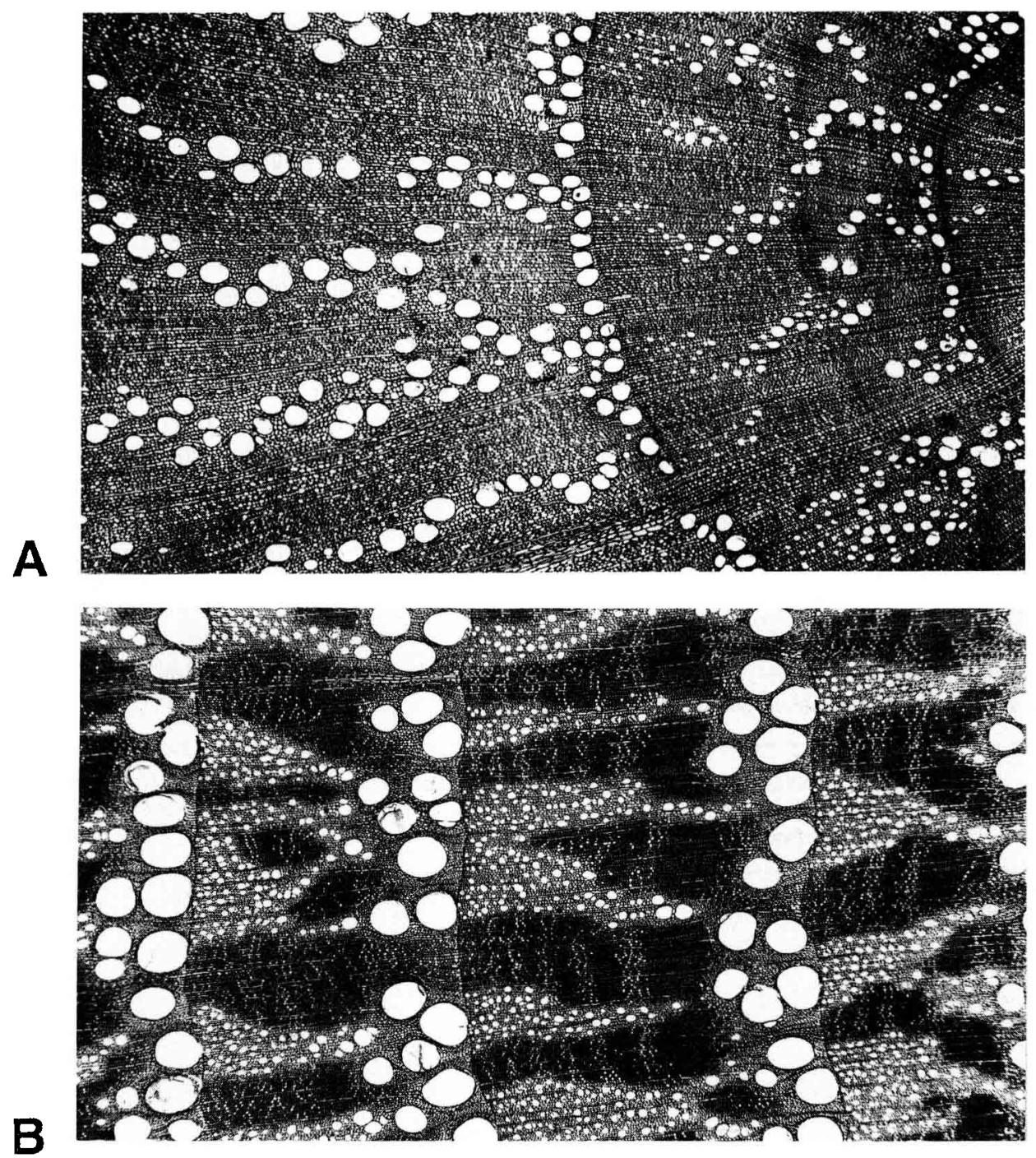

Fig 1. Plan ligneux de chêne (Quercus robur et/ou Quercus petraea) à l'état juvénile (en haut) et à l'état adulte (en bas). A) Répartition des vaisseaux dans les cernes d'âge 2 et 3 ans depuis la moelle. B) Répartition typique des vaisseaux dans les cernes de bois adulte. 
Pour cette raison, nous avons préféré mesurer la surface de ces éléments anatomiques plutôt qu'un diamètre, car la surface ne tient compte d'aucun paramètre de forme auquel la distinction entre les 2 variétés de chêne fait appel (Walker, 1978). Notre choix est conforté par les travaux de Savill et Mather (1988, communication personnelle) qui ne trouvent pas de différence au niveau de la surface des vaisseaux entre les 2 espèces de chêne.

\section{Données météorologiques}

Pour la période de 1960 à 1965 , nous avons utilisé les données de l'aéroport de NancyTomblaine, qui est situé à une vingtaine de kilomètres des forêts de Bride et de Saint-Jean; pour la période de 1966 à 1979 , nous avons retenu celles du Centre de recherches forestières de Champenoux.

Ne disposant pas de relevés pour une période commune aux 2 postes, nous n'avons pu les comparer. Cependant nous avons pensé que le climat différait peu entre les 2 localités: elles sont distantes de $10 \mathrm{~km}$ et situées à même altitude.

Les données dont nous disposions étaient : - les précipitations journalières exprimées en $\mathrm{mm}$;
- les températures maximales et minimales journalières exprimée en ${ }^{\circ} \mathrm{C}$.

Nous avons regroupé ces données en 53 périodes climatiques dont la durée variait de 2 à 14 mois.

\section{RÉSULTATS ET DISCUSSION}

\section{Expériences menées sur jeunes plants}

\section{Importance de la variabilité individuelle de la surface des premiers vaisseaux formés dans le bois initial}

Cette étude n'a été menée que sur les 5 plants du lot témoin afin d'éviter toute interférence avec les traitements de défoliaison.

Le tableau II qui présente les résultats de l'analyse de variance montre un effet de l'âge cambial hautement significatif.

Les différences de surfaces sont importantes : $5570 \mu \mathrm{m}^{2}$ la $1^{\text {re }}$ année, 16670 et $32340 \mu \mathrm{m}^{2}$ pour les $2^{\mathrm{e}}$ et $3^{\mathrm{e}}$ années (tableau IV).

Tableau I. Récapitulatif des différents points traités dans l'étude du déterminisme de la surface des vaisseaux du bois initial de $Q$ robur et $Q$ petraea.

\begin{tabular}{|c|c|c|c|c|c|c|c|c|c|c|}
\hline \multirow[b]{2}{*}{ Stades } & \multicolumn{2}{|c|}{$\begin{array}{l}\text { Importance de } \\
\text { la variabilité } \\
\text { individuelle }\end{array}$} & \multicolumn{2}{|c|}{$\begin{array}{l}\text { Effet de } \\
\text { l'appareil } \\
\text { foliaire }\end{array}$} & \multicolumn{2}{|c|}{$\begin{array}{c}\text { Effet de } \\
\text { l'age cambial }\end{array}$} & \multicolumn{2}{|c|}{$\begin{array}{l}\text { Effet de } \\
\text { la largeur } \\
\text { de cernes }\end{array}$} & \multicolumn{2}{|c|}{$\begin{array}{l}\text { Effet de } \\
\text { l'année } \\
\text { calendaire }\end{array}$} \\
\hline & $\mathrm{J}$ & $A$ & $\mathrm{~J}$ & $A$ & $\mathrm{~J}$ & $A$ & $\mathrm{~J}$ & $A$ & $\mathrm{~J}$ & A \\
\hline $\begin{array}{l}\text { Expériences sur } \\
\text { jeunes plants } \\
(Q \text { robur } L)\end{array}$ & + & - & + & - & + & - & + & - & - & - \\
\hline $\begin{array}{l}\text { Expérience sur } \\
\text { arbres adultes } \\
\text { ( } Q \text { robur } L \text { et } \\
Q \text { petraea Liebl) }\end{array}$ & + & + & - & - & + & - & + & + & - & + \\
\hline
\end{tabular}

+ : point traité ; - point non traité $; J=$ bois juvénile ; $A=$ bois adulte. 
Ces résultats confirment globalement ceux de Gasson (1984, communication personnelle) et de Savill et Mather (1988, communication personnelle). Ils montraient que, dans la phase juvénile, la taille des vaisseaux augmentait de façon très importante avec l'âge du cambium. Mais, contrairement aux résultats de Savill et Mather cités précédemment, nous n'avons pas trouvé d'effets dus à l'arbre.

Toutefois rappelons que nous n'avons travaillé que sur 5 arbres homogènes et que nous n'avons pris en compte qu'une partie de l'ensemble des vaisseaux du bois initial (ie ceux de la première rangée du bois de printemps).

\section{Importance de l'appareil foliaire sur la taille des premiers vaisseaux formés dans le bois initial}

Sur le tableau III, nous voyons que la surface des vaisseaux est sous la dépendance, à la fois de l'âge du cerne et du traitement appliqué.

Le tableau IV de comparaison de moyennes montre que :

- chez les plants totalement défoliés (traitement 1) la surface des vaisseaux aug- mente peu et de façon non significative avec l'âge du cerne, elle est comparable à celle rencontrée la 1re $^{\text {re }}$ année chez les plants des 2 autres lots ;

- l'absence de feuilles se marque sur la $3^{e}$ année des plants : la surface des vaisseaux des plants défoliés est significativement plus petite que celle des arbres intacts et que celle des arbres partiellement défoliés;

- la surface des vaisseaux est peu affectée par une ablation partielle du feuillage.

Nous pouvons donc penser que les vaisseaux des chênes ne répondent qu'à une modification très importante de la masse de leur feuillage.

Il faut remarquer, sans qu'aucune hypothèse ne soit émise, que dès la $2^{\mathrm{e}}$ année les arbres témoins avaient des vaisseaux de surface significativement plus importants que les arbres ayant subi une défoliaison, alors que celle-ci n'était intervenue qu'après la formation des vaisseaux. Une remarque analogue est faite dans l'étude de l'effet calendaire sur la taille des vaisseaux. II semblerait que des facteurs extérieurs aient un effet rétroactif sur le bois (Bazerque, 1974).

Tableau II. Effet de l'arbre et de l'âge cambial du cerne sur la surface des vaisseaux de la 1re rangée du bois initial chez le chêne pédonculé ( $Q$ robur).

\begin{tabular}{lcccc}
\hline $\begin{array}{l}\text { Source de } \\
\text { variation }\end{array}$ & $\begin{array}{c}\text { Somme des carrés } \\
\text { des écarts }\end{array}$ & $d d l$ & Carré moyen & $\begin{array}{c}F \\
\text { calculé }\end{array}$ \\
\hline Arbre & 133 & 4 & 33,4 & $1,5 \mathrm{NS}$ \\
$\begin{array}{l}\text { Age cambial } \\
\text { du cerne }\end{array}$ & 1809 & 2 & 904,5 & $41,7^{\star \star \star}$ \\
$\begin{array}{l}\text { Erreur } \\
\text { Total }\end{array}$ & 173 & 8 & 21,7 & \\
& 2116 & 14 & & \\
\hline
\end{tabular}

Analyse de variance portant sur les arbres témoins. La surface des vaisseaux est exprimée en $\mu m^{2}$. NS : test non significatif ; ${ }^{* \star *}$ : test significatif au seuil de $1 \%$. 
Tableau III. Effet des traitements de défoliaison, de l'âge du cerne et de l'interaction traitement $x$ âge du cerne sur la surface des vaisseaux (en $\mu \mathrm{m}^{2}$ ) de la $1^{\text {re }}$ rangée du bois initial chez le chêne pédonculé.

\begin{tabular}{lcccc}
\hline Source de variation & $\begin{array}{c}\text { Somme des carrés } \\
\text { des écarts }\end{array}$ & ddl & Carré moyen & F calculé \\
\hline Traitement & 689 & 2 & 344,3 & $20,8^{\star \star \star}$ \\
Age du cerne & 2244 & 2 & 1122,0 & $67,9^{\star \star \star}$ \\
$\begin{array}{l}\text { Interaction traitement } \\
\text { x âge du cerne }\end{array}$ & 829 & 4 & 207,3 & $12,5^{\star \star \star}$ \\
Variabilité individuelle & 595 & 36 & 16,6 & \\
Total & 4357 & 44 & 99,0 & \\
\hline
\end{tabular}

Analyse de variance sur l'ensemble de 15 plants. ${ }^{* * *}$ : test significatif au seuil de $1 \%$.

Tableau IV. Test de comparaison de moyennes de Newman-Keuls des combinaisons traitement $x$ âge cambial du cerne sur la surface des vaisseaux de la $1^{\text {re }}$ rangée du bois initial chez le chêne pédonculé ( $Q$ robur).

\begin{tabular}{llll}
\hline \multicolumn{1}{c}{ Facteurs } & $\begin{array}{l}\text { Surface moyenne } \\
\text { des vaisseaux } \\
(\text { Traitements }\end{array}$ & $\begin{array}{l}\text { Groupes } \\
\text { homogènes }\end{array}$ \\
\hline Témoin & $\begin{array}{l}\text { Age médullaire } \\
\text { du cerne }\end{array}$ & 32340 & $\mathrm{~A}$ \\
$1 / 2$ défoliaison & 3 & 27570 & $\mathrm{~A}$ \\
Témoin & 2 & 16670 & $\mathrm{~B}$ \\
Défoliaison totale & 3 & 10310 & $\mathrm{C}$ \\
$1 / 2$ défoliaison & 2 & 10150 & $\mathrm{C}$ \\
Défoliaison totale & 2 & 9110 & $\mathrm{C}$ \\
$1 / 2$ défoliaison & 1 & 6930 & $\mathrm{C}$ \\
Défoliaison totale & 1 & 6840 & $\mathrm{C}$ \\
Témoin & 1 & 5570 & $\mathrm{C}$ \\
\hline
\end{tabular}

Les surfaces des vaisseaux $\left(\mu \mathrm{m}^{2}\right)$ des combinaisons figurant devant la mème lettre ne diffèrent pas de façon significative ; seuil de signification : $5 \%$. 


\section{Expérience menée sur arbres adultes}

\section{Importance de la variabilité individuelle de la surface des vaisseaux du bois initial}

\section{Étude dans le bois juvénile}

La surface moyenne des vaisseaux de chaque arbre calculée sur 17 années est extrêmement variable d'un arbre à l'autre (fig 2).

Sur le tableau $V$, nous voyons que les surfaces des vaisseaux peuvent passer du simple au triple : $11164 \mu \mathrm{m}^{2}$ chez l'arbre ayant les plus petits vaisseaux contre $34988 \mu \mathrm{m}^{2}$ chez l'arbre présentant les vaisseaux les plus importants. C'est-à-dire, en admettant que les vaisseaux soient de section circulaire, un diamètre de $119 \mu \mathrm{m}$ à $211 \mu \mathrm{m}$.

L'analyse de variance présentée dans le tableau VI montre que la variabilité est contrôlée par l'arbre mais aussi par l'âge du cerne. Ces 2 effets expliquent respectivement 35 et $32 \%$ de la variabilité totale (tableau VIII).

La surface des vaisseaux du bois initial du côté juvénile apparait donc sous un contrôle assez étroit de l'individu, ce qui

Tableau V. Moyenne et variabilité entre arbres de la surface des vaisseaux du bois initial mesurés chez 37 chênes adultes $(Q$ robur et $Q$ petraea).

\begin{tabular}{lll}
\hline $\begin{array}{l}\text { Surface des } \\
\text { vaisseaux du } \\
\text { bois initial }\end{array}$ & $\begin{array}{l}\text { Moyenne } \\
\text { générale } \\
\left(\mu m^{2}\right)\end{array}$ & $\begin{array}{l}\text { Moyenne } \\
\text { d'arbres } \\
\text { extrêmes }\left(\mu m^{2}\right)\end{array}$ \\
\hline Bois juvénile & 22148 & $\begin{array}{l}11164 \\
34988\end{array}$ \\
Bois adulte & 57494 & 41610 \\
& & 81540
\end{tabular}

La moyenne est calculée sur 17 années pour le bois juvénile et sur 20 années, pour le bois adulte.

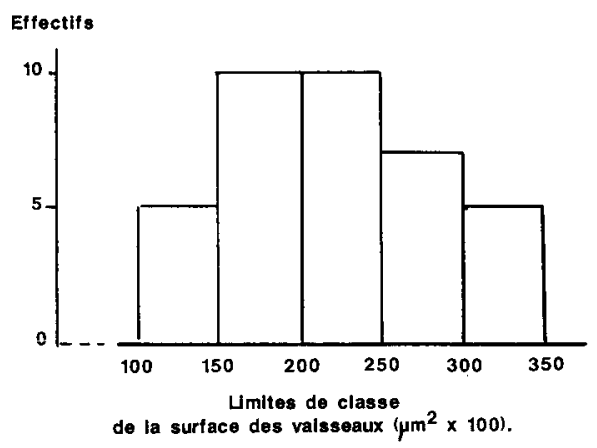

Fig 2. Histogramme de la répartition de la surface moyenne des vaisseaux du bois initial chez le chêne ( $Q$ robur et $Q$ petraea). Pour chaque arbre, la valeur est la moyenne de 17 années de bois juvénile des 37 arbres adultes.

est une condition nécessaire pour soupçonner un facteur génétique, et de l'âge du cerne puisque ces 2 facteurs à eux seuls représentent $67 \%$ de la variabilité totale. Ce pourcentage est un minimum, l'analyse de variance est une analyse à 2 facteurs sans répétition et l'effet de l'interaction individu $x$ année se trouve ainsi inclus dans la variance résiduelle.

II existe une relation linéaire $(r=0,983$, test significatif au seuil de 1\%o) entre la surface moyenne annuelle des vaisseaux et l'âge du cerne (fig 3 ).

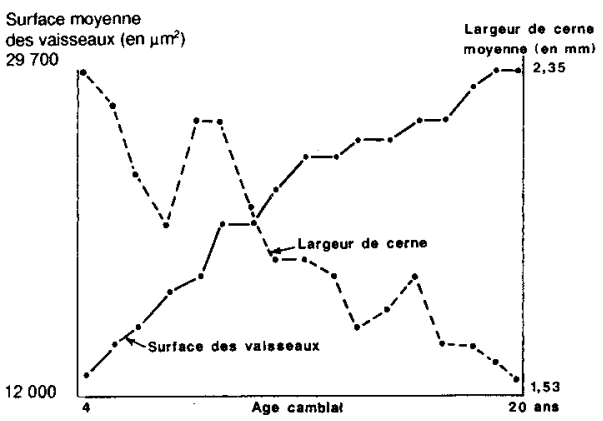

Fig 3. Variabilité de la surface moyenne annuelle des vaisseaux et de la largeur de cernes dans le bois juvénile des 37 chênes adultes $(Q$ robur et $Q$ petraea) en fonction de l'âge cambial des cernes. 
Tableau VI. Effet de l'arbre et de l'âge cambial du cerne sur la surface des vaisseaux $\left(\mu \mathrm{m}^{2}\right)$ du bois initial chez les chênes adultes ( $Q$ robur et $Q$ petraea).

\begin{tabular}{llccc}
\hline $\begin{array}{l}\text { Source de } \\
\text { variation }\end{array}$ & $\begin{array}{l}\text { Somme des carrés } \\
\text { des écarts }\end{array}$ & $d d l$ & Carré moyen & $\begin{array}{l}F \\
\text { calculé }\end{array}$ \\
\hline Arbre & 2073460 & 36 & 57596,1 & $19,4^{\star \star \star}$ \\
$\begin{array}{l}\text { Age cambial } \\
\text { du cerne }\end{array}$ & 1775047 & 16 & 110940,5 & $37,4^{* * *}$ \\
Erreur & 1709703 & 576 & 2968,2 & \\
Total & 5558210 & 628 & & \\
\hline
\end{tabular}

Analyse de variance portant sur le bois juvénile de 37 arbres et 17 années. *** : test significatif du seuil de $1 \%$.

Les résultats que nous avons obtenus sur des chênes adultes et sur de jeunes plants, en rappelant que chez ces derniers nous ne prenions en compte que la première rangée de vaisseaux, sont comparables à ceux obtenus par Gasson (1984, communication personnelle) et Savill et Mather (1988, communication personnelle). Ces auteurs montraient que l'augmentation de la taille des vaisseaux se produisait jusqu'à l'âge de cerne de 20 ans et parfois bien au-delà. Dans le peuple-

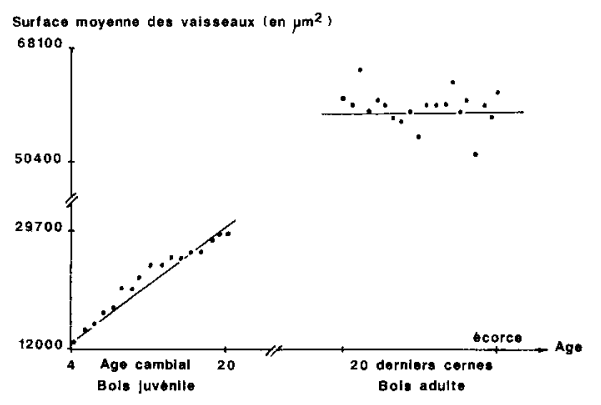

Fig 4. Effet de l'âge des cernes sur la surface des vaisseaux de leur bois initial chez le chêne ( $Q$ robur et $Q$ petraea). Ces valeurs sont la moyenne annuelle de 37 arbres, pour un âge cambial de 4 à 20 ans dans le bois juvénile et pour les 20 dernières années sous écorce pour le bois adulte. ment que nous avons étudié, la figure 4 montre d'ailleurs que les vaisseaux n'ont pas encore atteint leur "dimension adulte» à l'âge cambial de 20 ans puisque à cet âge la surface moyenne des vaisseaux est de $22148 \mu \mathrm{m}^{2}$ alors que, dans le bois situé au voisinage de l'écorce, elle est de $57494 \mu \mathrm{m}^{2}$ (tableau V).

\section{Dans le bois adulte}

La surface moyenne des vaisseaux de chaque arbre, calculée sur 20 ans, est elle aussi extrêmement variable entre les individus (fig 5).

Sur le tableau $V$, nous voyons que cette valeur peut passer du simple au double pour les arbres extrêmes.

La variabilité que nous présente le tableau VII est due pour $60 \%$ à l'effet de l'arbre et $4 \%$ seulement à l'effet de l'année calendaire (tableau VIII).

La part de l'effet arbre est ici importante, il paraît difficile de ne l'attribuer qu'à l'absence de distinction entre les 2 espèces de chêne (Savill et Mather, 1988, communication personnelle).

II faut aussi remarquer que l'effet de l'arbre est plus important dans le bois 
Tableau VII. Effet de l'arbre et de l'année calendaire sur la surface des vaisseaux $\left(\mu \mathrm{m}^{2}\right)$ du bois initial chez les chènes adultes ( $Q$ robur et $Q$ petraea).

\begin{tabular}{lrrrr}
\hline $\begin{array}{l}\text { Source de } \\
\text { variation }\end{array}$ & $\begin{array}{l}\text { Somme des carrés } \\
\text { des écarts }\end{array}$ & ddl & Carré moyen & $\begin{array}{l}F \\
\text { calculé }\end{array}$ \\
\hline Arbre & 7304872 & 36 & 202913,1 & $34,0^{* * *}$ \\
Année calendaire & 518349 & 19 & 27281,5 & $4,6^{* * *}$ \\
Erreur & 4079887 & 684 & 5964,8 & \\
Total & 11903108 & 739 & 16107,1 & \\
\hline
\end{tabular}

Analyse de variance sur le bois adulte de 37 arbres et 20 années. *** : test significatif au seuil de $1 \%$.

adulte que dans le bois juvénile: nous constatons en effet que dans ce dernier cas la part de variabilité due à l'arbre est de $35 \%$ contre $60 \%$ dans le bois adulte.

\section{Effet de la largeur de cerne sur la surface des vaisseaux du bois initial}

L'étude de la corrélation entre la surface des vaisseaux et la largeur des cernes a été réalisée à “différents niveaux», que nous définissons ci-dessous.

- Niveau arbre : nous avons calculé la corrélation entre la surface moyenne des vaisseaux et la largeur de cerne moyenne calculée pour chaque arbre. Cette corrélation

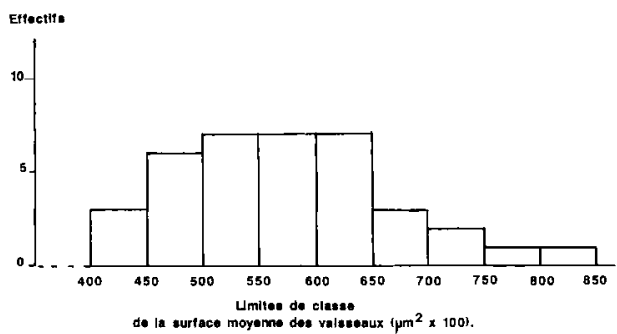

Fig 5. Histogramme de la répartition de la surface moyenne des vaisseaux du bois initial chez le chêne ( $Q$ robur et $Q$ petraea). Pour chaque arbre, la valeur est la moyenne de 20 années de bois adulte des 37 arbres adultes. nous permet de savoir si la vitesse moyenne de croissance en diamètre d'un individu est lié à la surface moyenne de ses vaisseaux.

Dans le bois juvénile comme dans le bois adulte, nous avons obtenu un coefficient $r$ calculé sur 37 couples.

- Niveau "âge" (bois juvénile) ou "calendaire" (bois adulte): nous avons calculé la corrélation entre la moyenne des surfaces des vaisseaux et la moyenne des largeurs de cernes calculées âge par âge dans le bois juvénile ou année calendaire par année calendaire dans le bois adulte.

Tableau VIII. Pourcentage explicatif des différentes sources de variation de la surface des vaisseaux chez les chênes adultes $(Q$ robur et $Q$ petraea).

\begin{tabular}{lccc}
\hline & \multicolumn{2}{c}{ Source de variation } \\
\cline { 2 - 3 } $\begin{array}{l}\text { Matériel } \\
\text { végétal }\end{array}$ & Arbre & $\begin{array}{l}\text { Age } \\
\text { cambial } \\
\text { du cerne }\end{array}$ & $\begin{array}{c}\text { Année } \\
\text { calendaire }\end{array}$ \\
\hline
\end{tabular}

\begin{tabular}{llll} 
Bois juvénile & $35 \%$ & $32 \%$ & - \\
Bois adulte & $60 \%$ & - & $4 \%$ \\
\hline
\end{tabular}


Dans le bois juvénile nous disposions donc de 17 couples et de 20 couples dans le bois adulte.

- Niveau "années calendaires décalées» pour le bois adulte uniquement: les vaisseaux du bois initial de l'année $n$ se mettent en place, en absence de toute feuille, grâce aux réserves emmagasinées par l'arbre au cours de l'année de végétation précédente soit l'année $n-1$ (Wareing, 1951). Pour cette raison la corrélation a été calculée sur les données décrites comme ci-dessus, mais la corrélation a été calculée entre la largeur de cerne de l'année $n-1$ et la surface des vaisseaux de l'année $n$ soit 19 couples de données.

Les corrélations largeur de cernesurface des vaisseaux au niveau "année» ou "âge" peuvent nous indiquer si les conditions climatiques (effet "année calendaire") ou l'âge depuis la moelle ont une influence favorable ou défavorable sur la surface des vaisseaux d'un cerne à l'autre à l'intérieur d'un individu.

Dans le bois juvénile

Le tableau IX montre que dans le bois juvénile les individus à croissance rapide ne sont pas ceux présentant des vaisseaux spécialement gros ou petits.

Nous constatons que, si nous nous plaçons au niveau "âge", la surface des vaisseaux est corrélée de façon significative et négative à la largeur de cerne moyenne (fig 3). Lorsque la largeur de cerne augmente, la surface des vaisseaux diminue de manière très nette.

Il faut remarquer que la surface moyenne des vaisseaux augmente avec l'âge cambial quelle que soit la fourchette dans laquelle évolue la largeur de cerne (fig 6a et b).

\section{Dans le bois adulte}

Le tableau IX montre que, dans le bois adulte, la largeur des cernes et la surface des vaisseaux du bois initial sont 2 paramètres indépendants tant au niveau "arbre» qu'au niveau "calendaire» ; c'est un résultat analogue à celui qu'avait trouvé Savill (1986) au niveau «calendaire».

II vaut d'être noté que nous n'avons pas non plus mis en évidence de corrélation entre la surface des vaisseaux de l'année $n$ et la largeur de cerne de l'année $n-1$

Tableau IX. Coefficient de corrélations entre la surface des vaisseaux du bois initial et la largeur de cerne chez les 37 chênes adultes ( $Q$ robur et $Q$ petraea).

\begin{tabular}{llllll}
\hline Niveau étudié & \multicolumn{2}{c}{ Bois juvénile } & & & \multicolumn{2}{c}{ Bois adulte } \\
\cline { 2 - 3 } & $\begin{array}{l}\text { Coefficient de } \\
\text { corrélation r }\end{array}$ & $d d l$ & & $\begin{array}{l}\text { Coefficient de } \\
\text { corrélation r }\end{array}$ & $d d l$ \\
\hline Arbre & $-0,186 \mathrm{NS}$ & 35 & & $-0,132 \mathrm{NS}$ & 35 \\
$\begin{array}{l}\text { Age cambial (bois } \\
\text { juvénile) ou année } \\
\text { calendaire (bois adulte) }\end{array}$ & $-0,927^{* * *}$ & 15 & & $0,004 \mathrm{NS}$ & 18 \\
Année décalée & - & - & & $-0,137 \mathrm{NS}$ & 17 \\
\hline
\end{tabular}

${ }^{* * *}$ : test significatif au seuil de $1 \%$; NS : test non significatif. 


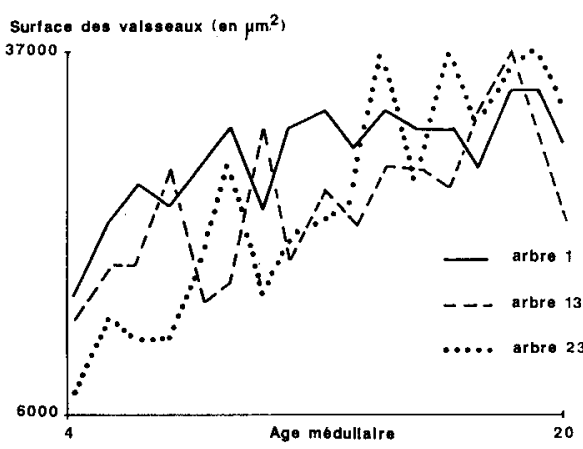

A

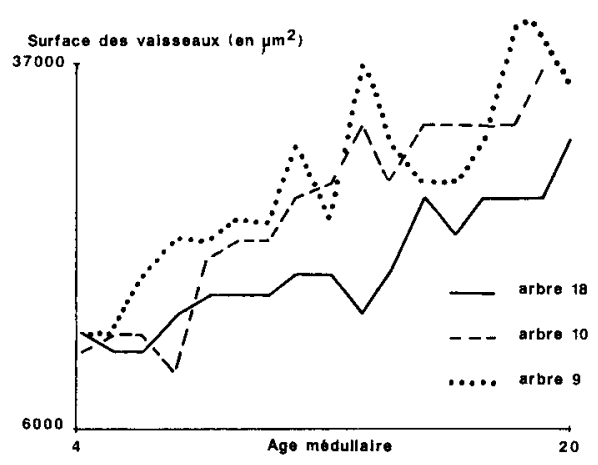

B

Fig 6. Evolution de la surface moyenne des vaisseaux dans le bois juvénile chez des chênes ( $Q$ robur et $Q$ petraea) de croissance annuelle différente. A. Chênes à croissance annuelle importante et très variable d'année en année, comprise entre 1,14 et $3,50 \mathrm{~mm}$. B. Chênes à croissance annuelle faible et peu variable d'année en année, comprise entre 0,67 et $2,61 \mathrm{~mm}$.

comme pouvaient le laisser supposer les travaux de Wareing (1951).

\section{Effet de l'année calendaire sur la taille des vaisseaux du bois initial dans le bois adulte}

Le tableau VIII nous avait indiqué que l'effet de l'année, bien que n'expliquant que $4 \%$ de la variabilité totale de la surface des vaisseaux du bois initial (contre 60\% pour l'effet arbre), était significatif. Nous avons cherché les facteurs climatiques qui pouvaient expliquer ces effets (discrets) de l'année calendaire.

Cette étude, qui est différente de celle de l'effet de l'âge cambial n'a été réalisée que sur le bois adulte (tableau I).

En toute rigueur, nous devons considérer que, dans notre cas, l'effet de l'année est un effet résultant de la combinaison de f'influence du climat et des traitements sylvicoles.

Dans notre étude nous n'avons pas pu différencier ces 2 effets. Toutefois, les arbres provenant de 6 parcelles différentes, nous pouvons considérer que des effets systématiques de la sylviculture ne sont pas à craindre pour expliquer l'effet calendaire.

En revanche, une influence du climat sur la surface des vaisseaux peut être soupçonnée.

Le climat auquel nous avons à faire est un climat semi-océanique, où la moyenne des précipitations était, pour la période étudiée, de $733 \mathrm{~mm}$ d'eau par an, la moyenne des températures maximales de $11,4^{\circ} \mathrm{C}$ et des températures minimales de $1,1^{\circ} \mathrm{C}$.

Les précipitations étaient réparties uniformément sur l'ensemble des mois de l'année. II faut noter 2 années plutôt sèches : 1964 et 1976 et 2 années humides : 1970 et 1979.

Entre le mois d'août de l'année $n-1$ et le mois de juin de l'année $n$, nous avons procédé à 54 regroupements qui allaient de 1 à 14 mois pour chacune des 3 données climatiques suivantes : la température maxi- 
male, la température minimale et les précipitations.

Nous avons ensuite calculé les coefficients de corrélation entre la surface des vaisseaux de l'année $n$ de l'arbre moyen et la moyenne des données climatiques regroupées, c'est ainsi 162 coefficients $r$ qui ont été calculés (54 regroupements $\times 3$ données climatiques).

Les corrélations entre les paramètres du climat et la surface des vaisseaux ne sont pas significatives à l'exception d'une seule qui lie la surface des vaisseaux aux températures maximales de la période de septembre à décembre de l'année précédant leur formation $(r=0,530$, ddl 17 , seuil de signification $5 \%$ ) : aux périodes froides de septembre à décembre de l'année $n-1$, semblent correspondre des vaisseaux de surface un peu plus faible (ceci est relativement bien apparent pour 1969).

Nos résultats sont différents de ceux de Eckstein et Frisse (1979) qui écrivaient que la variation de la taille des vaisseaux était influencée par les précipitations.

Au regard de la figure 7 , la taille des vaisseaux en 1976 est difficilement explicable par la moyenne des températures maximales de septembre à décembre de l'année précédente qui n'était pas particulièrement basse.

Cependant Savill et Mather (1988, communication personnelle) montrent que, certes, l'effet du climat influe sur la taille des vaisseaux, mais qu'en 1976, malgré la sécheresse enregistrée aussi en GrandeBretagne, les vaisseaux ont une taille peu différente de ceux des autres années.

Dans notre étude nous ne pouvons peut-être pas attribuer cette faible surface des vaisseaux à la sécheresse, car elle s'est produite en été lorsque les vaisseaux étaient déjà formés. (II faut rapprocher cette observation de celle faite au chapitre «Importance de l'appareil foliaire sur la

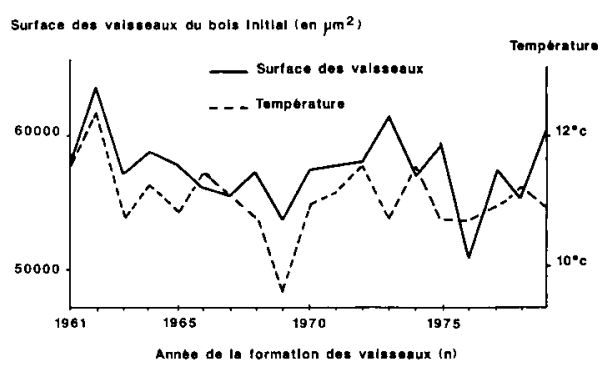

Fig 7. Surface des vaisseaux et température maximale moyenne de la période de septembre à décembre de l'année précédant la formation des vaisseaux en fonction du temps.

taille des premiers vaisseaux formés dans le bois initial».)

\section{CONCLUSION}

L'objectif de cette étude était de mieux connaître le déterminisme de la surface des vaisseaux du bois initial des chênes indigènes ( $Q$ robur $L$ et $Q$ petraea Liebl).

Ces éléments, assez peu étudiés jusqu'ici, avaient en effet été remarqués comme influençant certaines propriétés technologiques de ce bois. En mieux connaître le déterminisme était donc une condition nécessaire pour espérer mieux les maîtriser.

À la lumière de ces travaux, il apparaît que la surface des vaisseaux est peu modifiée par l'environnement.

Sur les plants de 3 ans, seule l'ablation totale des feuilles a une influence significative sur leur surface ; chez les arbres adultes seules les températures automnales agissent sur ces éléments, mais de manière peu significative.

Cependant la surface des vaisseaux ne demeure pas constante tout au long de la vie de l'arbre. Elle évolue très sensible- 
ment en fonction de l'âge cambial dans la phase juvénile de l'arbre. L'évolution est sans doute due à une programmation endogène difficile à contrecarrer.

Une remarque s'impose et c'est un point important qui ouvre le plus d'espoir en matière de sélection : la variabilité individuelle (entre arbres) est tout à fait considérable jusque et y compris dans le bois juvénile.

Nous allons poursuivre cette étude en nous intéressant à la relation entre la surface des vaisseaux du bois adulte et du bois juvénile, afin de voir si une sélection juvénile est possible et à quel âge minimum celle-ci peut intervenir.

Des travaux similaires, déterminisme et liaison juvénile adulte, seront aussi présentés au sujet d'autres caractéristiques anatomiques.

\section{REMERCIEMENTS}

Ce travail a été réalisé avec la collaboration technique d'É Varcin et $P$ Gelhaye que je remercie.

\section{RÉFÉRENCES}

Aubert M (1987) Recherche de relations entre caractéristiques simples du bois de chêne rouge (Quercus rubra $L$ ) mesurées par voie non destructive et deux de ses propriétés technologiques : stabilité dimensionnelle des planches et qualité des placages d'ébénisterie. Doctorat de $3^{e}$ cycle en Sciences du bois, université de Nancy I, UER PhysiqueChimie-Biologie, INRA, station de recherches sur la qualité des bois

Bazerque F (1974) Étude dendrochronologique et dendroclimatologique de deux peuplements de pins sylvestres. Thèse de spécialité, université de Nancy I, UER Physique, Chimie, Biologie

Cinotti B (1990) La gélivure des chênes facteurs prédisposants individuels et mécanique du phénomène. Rev For Fr 2, 145-147
Eckstein D, Frisse E (1979) Environmental influences on the vessel size of Beech and Oak. IAWA Bull 2/3, 36-37

Flechter JM (1975) Relation of abnormal earlywood in oak to dendrochronology and climatology. Nature 254, 506-507

Gasson P (1984) Recognition of characteristic features of secondary xylem of selected hardwoods. Thesis, Univ London, Imperial College

Gasson P (1987) Some implications of anatomical variations in the wood of Pedunculate oak (Quercus robur $\mathrm{L}$ ) including comparisons with Common beech (Fagus silvatica $\mathrm{L}$ ). IAWA vol $8, n^{\circ} 2,149-166$

Gradwell GR (1974) The effects of defoliations on tree growth. The British Oak (MG and FH Perrings, eds) $182-193$

Hofmann P (1987) Mesure de la variabilité de la couleur du bois : méthodologie et influence de l'anatomie du plan ligneux et des variations angulaires du débit. DEA Sciences du Bois, université de Nancy I, octobre 1987

Huber $F$ (1982) Effet de défoliaisons des chênes par les hannetons sur la structure du bois. Rev For Fr XXXIV, 3-82

Kanowski PJ, Mather RA, Savill PS (1991) Short note: genetic control of oak shake, some preliminary results. Silvae Genet 40, 3/4, 166-168

Malphettes CB (1990) Les défoliateurs du chêne. Rev For Fr XLII, 2, 191-197

Marchal R (1983) Intérêt de la prise en compte de caractéristiques physiques et anatomiques simples du bois le chêne pour l'appréciation de la qualité des placages d'ébénisterie. DEA Sciences du Bois, université de Nancy 1

Pechmann HV, Aufsess HV (1973) Struktur und Farbe als Qualitätsmerkmale von Eichen Furnierholz. Holzforschung 27, 52-59

Polge $H$ (1966) Établissement des courbes de variation de la densité du bois par exploration densitométrique de radiographies d'échantillons prélevés à la tarière sur des arbres vivants. Applications dans les domaines technologique et physiologique. Thèse de doctorat en Sciences appliquées, faculté des sciences de l'université de Nancy

Polge H, Keller R (1973) Qualité du bois et largeur d'accroissements en forêt de Tronçais. Ann Sci For 30 (2), 91-126 
Polge $H$ (1984) Production de chênes de qualité en France. $\mathrm{N}^{\circ}$ spécial Rev For Fr

Savill PS (1986) Anatomical characters in the wood of Oak (Quercus robur $L$ and Quercus petraea Liebl) which predispose trees to shake. Commonw For Rev 65 (2), 109-116

Walker FS (1978) Pedunculate and Sessile oaks: species determination from differences between their wood. National Maritime Museum, Greenwich, Archeological Series $N^{\circ} 4$, Research Laboratory for Archeology and History of Act, Oxford University, Publ n ${ }^{\circ} 2$, BAR International Series 51, 329-338

Wareing PF (1951) Growth studies in woody species. 4. The initiation of cambial activity in ring-porous species. Physiol Plant 4, 546-562 